\title{
Die Purinkörper der menschlichen Faeces.
}

Von

M. Krüger und A. Schittenhelm.

(Aus der medicinischen Klinik der Universität Breslau.)

(Der Redaction zugegangen am 5. März 1902.)

Ueber die Ausscheidung von Harnsäure und Purinbasen mit den menschlichen Excrementen hat in eingehender Weise zuerst Weintraud Untersuchungen angestellt, deren Resultate er in zwei Abhandlungen, einer vorläufigen Mittheilung im Centralblatt für innere Medicin 1895, S. 433, und später in einem ausführlicheren Vortrage in den Verhandlungen des Congresses für innere Medicin 1896 mitgetheilt hat.

Der Gedanke, menschliche Faeces auf das Vorkommen von Purinbasen hin zu untersuchen, kam Weintraud bei der Beobachtung eines Falles von Leukämie, bei welchem trotz starker Vermehrung der Leucocyten nur eine geringe Steigerung der Harnsäureausscheidung im Harne zu constatiren war. Thatsächlich fand er im Kothe des Leukämischen die zehnfache Menge (etwa $1 \mathrm{~g}$ ) an Purinbasen - aus dem Stickstoffgehalte des Kupferoxydulniederschlages auf Hypoxanthin umgerechnet -, als ein normaler Mensch während derselben Zeit mit dem Harne entleert. Aber nicht nur beim Leukämiker, sondern auch im Kothe anderer Patienten und in normalen Faeces sind Purinbasen stets vorhanden, in letzteren $0,1-0,5 \mathrm{~g}$ pro die, sodass sie Weintraud ganz allgemein als Bestandtheile der menschlichen Faeces bezeichnen kann.

Was die Herkunft dieser Basen betrifft, so hat Weintraud durch eine Reihe von Versuchen die Nahrung als Quelle derselben mit Sicherheit ausgeschlossen. Denn er zeigt, dass 
nucleinreiche Kost auf die Ausscheidung der Purinbasen im Kothe keinen Einfluss hat, woraus auf eine völlige Resorption der die Basen enthaltenden Eiweisskörper geschlossen werden muss. Ferner konnte Weintraud die Basen im Kothe auch dann nachweisen, wenn vollkommen stickstofffreie Kost gegeben wurde oder wenn die Nahrung nur aus Milch bestand, obwohl das Nuclein der Milch keine Purinkörper enthält. Da endlich die Basen auch im Meconium eines Kindes, welches mit Atresia ani geboren war, gefunden wurden, so sind sie sicher nicht als unresorbirte Nahrungsreste aufzufassen, sondern *als ein Bestandtheil, der von der Darmwand oder von den grossen Verdauungsdrüsen, die ihr Secret in den Darm entleeren, herstammt».

Ueber die Zusammensetzung des Basengemenges in den Faeces gibt Weintraud in seiner vorläufigen Mittheilung an, dass es im Wesentlichen aus Hypoxanthin zu bestehen scheint, nach seinem Vortrage aber sind die drei Nucleinbasen, Xanthin, Hypoxanthin und Guanin, darin vorhanden.

Nach Weintraud hat sich Karl Petrén ${ }^{1}$ ) mit dem gleichen Thema beschäftigt, indem er, von denselben Fragen, wie sein Vorgänger ausgehend, dessen Versuche einer Nachprüfung mit einer anderen Methode der Basenfällung unterzieht. Die von Petrén erhaltenen Resultate wichen in einigen Punkten von denen Weintraud's ab. Nach letzterem enthält das Nucleoalbumin der (menschlichen) Galle Purinbasen, womit die Herkunft wenigstens eines Theiles der Kothbasen ihre Erklärung findet. Denn dass nicht die Gesammtmenge aus der Galle stammt, konnte Weintraud durch Analyse der gallenarmen Faeces eines Gelbsüchtigen nachweisen, in welchen gleichfalls Basen gefunden wurden.

Petrén untersuchte Rindergalle und fand weder in der Galle selbst noch in deren Nucleoalbumin Purinbasen. Indem er nun die an diesem Material erhaltenen Resultate auf mensch-

1) Die im skandinavischen Archiv für Physiologie 8, 315 und 9, 412 plublicirten Originalarbeiten standen uns leider nicht zur Verfügung; die mitgetheilten Angaben haben wir den ausführlichen Referaten in Maly's Thierchemie entnommen. 
liche Galle überträgt, kommt er nothwendig zu dem Schlusse, dass die Gallenblase nicht als Quelle der Faecesbasen anzusehen ist.

Die Menge der pro Tag mit den Excrementen ausgeschiedenen Basen wird von Weintraud und Petrén sehr verschieden angegeben. Nach jenem beträgt dieselbe beim normalen Menschen, wie schon oben erwähnt, $0,1-0,5 \mathrm{~g}$; Petrén dagegen findet nur $0,05-0,1 \mathrm{~g}$, indem er nach Salkowski für $1 \mathrm{~g}$ Silber in der Silberfällung $0,7381 \mathrm{~g}$ Basen rechnet. Diese Differenzen finden ihre Erklärung darin, dass die Autoren sich nicht derselben Fällungsmethode bei der Isolirung der Basen bedient haben. Weintraud wendet die Kupferoxydulmethode an; da aber Kupfersulfat und Bisulfit neben Purinkörpern auch andere Substanzen fällen, so dürften seine Zahlen zu hoch ausgefallen sein. Jedenfalls kommen sie aber, wie unser Versuch zeigt, den wahren Werthen sehr nahe.

Die von Petrén bevorzugte Methode der Fällung mit ammoniakalischer Silberlösung hat den übleren und wohl kaum zu beseitigenden Nachtheil, dass sie bei Anwesenheit einer ganzen Reihe von zum Theil bekannten Körpern die Purinbasen überhaupt nicht oder nur zum Theil niederschlägt. Solche die Fällung verhindernden Verbindungen finden sich aber gerade in den durch Kochen mit Säuren aufgeschlossenen Organen. Dasselbe gilt für Faeces; die mit Hülfe der Silbermethode aus-geführten quantitativen Bestimmungen können daher erst dann Anspruch auf Genauigkeit machen, wenn die Ergebnisse mit anderen Methoden kontrollirt und als richtig befunden sind.

Hiermit sind im Wesentlichen die Resultate der bisherigen Untersuchungen über Purinkörper in den Faeces mitgetheilt. Stoffwechselversuche mit Purinbasen, welche in unserer Klinik ausgeführt sind, führten uns auf dasselbe Arbeitsgebiet. . Durch diese Experimente ${ }^{1}$ ) war der Nachweis erbracht, dass in derselben Weise wie Hypoxanthin auch die Basen Adenin, Xanthin und wahrscheinlich auch Guanin, dem menschlichen Organismus einverleibt, in Harnsäure übergehen. Um diesen Vorgang

1) Diese Zeitschr., Bd. XXXIV, S. 549 . 
weiterhin in quantitativer Hinsicht verfolgen $\mathrm{zu}$ können, ist die Bestimmung der Resorptionsgrösse der einzelnen Basen, das ist des mit den Faeces unverändert ausgeschiedenen Theiles derselben nothwendig, was wiederum die Kenntniss der normal in den Excrementen enthaltenen Basenmenge und ferner auch die Art und die Mengenverhältnisse dieser Basen voraussetzt.

Obwohl Weintraud mit der Frage nach der Art der Basen, wie oben erwähnt, sich wohl beschäftigt hat und das Gemisch derselben als aus Xanthin, Hypoxanthin und Guanin bestehend annimmt, glaubten wir dennoch gerade diesen Theil seiner Untersuchungen einer Nachprüfung unterziehen zu müssen, nicht sowohl deshalb, weil man in seinen Mittheilungen Angaben über Trennungsmethoden, Analysen und Mengenverhältnisse der Basen überhaupt vermisst, als weil der vierten der Nucleinbasen, des Adenins, nirgends Erwähnung geschieht.

Da dieser unter allen natürlich vorkommenden Purinbasen am leichtesten nachweisbare Körper, welcher durch ein in schwerlöslichen, glänzenden Nadeln von bestimmtem Zersetzungspunkte krystallisirendes Pikrat charakterisirt ist, bei einer Untersuchung kaum entgehen kann, so ist nur anzunehmen, dass er thatsächlich unter den Kothbasen nicht vorkommt. Andererseits aber muss er, wenn die Ansicht Weintraud's über die Herkunft dieser Basen aus den in den Darm sich ergiessenden Secreten und aus der Darmschleimhaut richtig ist, ursprünglich in das Darmlumen mitausgeschieden sein, da in allen Organen, Geweben und Secreten, die bishe? auf Purinkörper untersucht sind, das Adenin stets in Gemcinschaft mit den übrigen Nucleinbasen gefunden ist.

Es kann daher nur beim Passiren des Darmkanals verschwunden sein. Eine Erklärung hierfür geben die Versuche von Schindler ${ }^{1}$ ) über das Verhalten der Nucleinbasen gegen Fäulnissbacterien. Die Fäulniss zeigt bemerkenswerther Weise dieselbe Wirkung auf Adenin, wie salpetrige Säure, indem sie es unter Ersatz der Aminogruppe durch Hydroxyl in Hypoxanthin überführt.

1) Diese Zeitschr., Bd. XIII, S. 439. 
Wenn somit als Grund für das Fehlen des Adenins unter den Kothbasen in ungezwungener Weise die Fäulniss anzusehen ist, so bedarf andererseits wieder das Vorkommen von Guanin einer Erklärung. Denn nach Schindler erleidet diese Base unter dem Einflusse der Fäulnissbacterien in ganz analoger Weise wie Adenin eine Umwandlung in Xanthin.

Die Anwesenheit von Guanin und das Fehlen von Adenin . unter den Basen des Kothes stellen daher einen Widerspruch in den Resultaten Weintraud's dar, der nur durch Nachprüfung seiner Versuche beseitigt werden kann.

Fxperimenteller Theil.

Um möglichst grosse Mengen der Basen zu erhalten (sodass der sichere Nachweis der einzelnen Bestandtheile des Gemisches keine Schwierigkeiten machen konnte), wurden die Faeces unserer Versuchsperson, welche gemischte klinische Kost mit Ausnahme von Kaffee, Cacao erhielt, während eines Zeitraumes von sechs Wochen gesammelt und in der folgenden Weise verarbeitet:

Die Tagesmenge an Koth wurde mit 2 Litern Wasser und $15 \mathrm{ccm}$. concentrirter Schwefelsäure mehrere Stunden (2-3) über freiem Feuer erhitzt. Die Filtrate wurden in den ersten Tagen nach Neutralisation mit Natronlauge mit je $50 \mathrm{ccm}$. $10 \%$ iger Natronlauge und 10\% iger Natriumcarbonatlösung versetzt, dann mit $3 \mathrm{~g}$ Ferrosulfat längere Zeit in der Wärme digerirt, um wenigstens einen Theil der Farbstoffe zu entfernen. Nach nochmaligem Filtriren wurden die Purinkörper aus der siedend heissen Lösung durch Kupfersulfat und Bisulfit gefällt.

Späterhin wurde auf die Entfärbung der einzelnen schwefelsauren Auskochungen verzichtet, da sich herausgestellt hatte, dass bei den Kupferfällungen die Hauptmenge der Farbstoffe in Lösung bleibt. Die schwefelsauren Filtrate wurden nunmehr zur Vorbereitung für die Fällung mit Kupfersulfat und Bisulfit mit Natronlauge alkalisch, dann mit Essigsäure stark sauer gemacht und nach dem Erhitzen noch einmal filtrirt. 
Die gut ausgewaschenen, vereinigten Kupferoxydulniederschläge wurden dann, in heissem Wasser suspendirt, durch Schwefelwasserstoff zersetzt. Nachdem noch Schwefelsäure hinzugefügt war, um etwa ausgeschiedenes Guanin in Lösung zu bringen, wurde vom Schwefelkupfer abfiltrirt. Da das Filtrat noch stark gefärbt war, wurde es in der Wärme so lange mit basischem Bleiacetat behandelt, bis die über dem Niederschlage stehende Flüssigkeit nur noch eine hellgelbe Farbe zeigte. Das Filtrat wurde zunächst mit Natronlauge alkalisch gemacht, dann mit $100 \mathrm{ccm}$. Natriumbisulfitlösung versetzt und vom entstandenen Niederschlage wiederum durch Filtriren befreit. Aus der auf diese Weise erhaltenen Lösung wurden die Basen nochmals durch Hinzufügen von Kupfersulfat ausgefällt.

Die Lösung der aus diesem Niederschlage isolirten Basen war nur noch wenig gefärbt und bereitete bei der Isolirung der Basen keine Schwierigkeiten. Sie wurde bis auf etwa $150 \mathrm{ccm}$. eingedampft und in der Wärme mit $30 \mathrm{ccm} .10 \%$ igen Ammoniaks im Ueberschuss versetzt. Der sofort entstandene Niederschlag von Guanin wurde nach 24 stündigem Stehen abfiltrirt, noch einmal mit 2\% igem Ammoniak in der Wärme digerirt, nach weiteren 24 Stunden wiederum abfiltrirt und endlich zur Reinigung in verdünnter Natronlauge gelöst und durch Ansäuern der alkalischen Lösung mit Essigsäure wieder ausgefällt. Erhalten wurde 2,363 g Guanin.

Die Prüfung des Guanins auf beigemengtes Epiguanin (7-Methylguanin) ergab ein negatives Resultat. Zum Naclweise des Epiguanins wurde das Guanin längere Zeit mit etwa 1\% iger Essigsäure ausgekocht. Aus dem Filtrate schied sich beim Erkalten ein geringer amorpher Niederschlag aus, der beim Einengen der Flüssigkeit nur wenig vermehrt wurde. De Prismen des Epiguanins waren in ihm nicht nachzuweiser, er gab das in rhombischen oder sechsseitigen Blättchen krystallisirende Pikrat des Guanins.

Die Hauptmenge des Guanins wurde zur Identificirung $n$ das in langen Prismen krystallisirende Sulfat übergeführt, dessm Analyse folgende Zahlen ergab: 
$0,1268 \mathrm{~g}$ Substanz: $0,0106 \mathrm{~g} \mathrm{H}_{2} \mathrm{O}$ bei $140^{\circ} ; 29,00 \mathrm{ccm}$.

1/10 N Oxalsäure nach $\mathrm{Kjeldahl.}$

$0,3060 \mathrm{~g}$ Substanz: 0,1624 g $\mathrm{BaSO}_{4}$.

$\left(\mathrm{C}_{5} \mathrm{H}_{5} \mathrm{~N}_{5} \mathrm{O}\right)_{2} \cdot \mathrm{H}_{2} \mathrm{SO}_{4}+2 \mathrm{H}_{2} \mathrm{O}$ :

Verl.: $8,26 \% \mathrm{H}_{2} \mathrm{O} ; 32,11 \% \mathrm{~N} ; 22,48 \% \mathrm{H}_{2} \mathrm{SO}_{4}$.

Gef.: $8,36 \%$ > $32,02 \%$ > $22,32 \%$ »

Die ammoniakalischen Filtrate vom Guanin wurden nach Entfernung des Ammoniaks mit Salzsäure zur Trockne eingedampft und der Rückstand zur Beseitigung der überschüssigen Salzsäure noch einmal mit Wasser, dann mehrere Male mit Alkohol eingetrocknet.

Bei der Verarbeitung des nunmehr aus den salzsauren Salzen der Basen bestehenden Gemenges nach dem Trennungsverfahren von Krüger und Salomon ${ }^{1}$ ) zeigte es sich, dass aus der Lösung der Salze in wenig Wasser, selbst nach mehrstündigem Stehen im Eisschranke, sich kein Xanthin ausschied, obwohl die weitere Untersuchung das Vorhandensein desselben ergab.

Die gleiche Beobachtung wurde in allen Fällen gemacht, wo Xanthin nur in untergeordneter Menge neben grösseren Mengen anderer Basen, namentlich Adenins, vorkommt. Dann ist die Methode von Krüger und Salomon dahin abzuändern, dass man vor der Isolirung des Xanthins erst das Adenin, resp. die anderen störenden Basen herausschafft.

In dieser Weise wurde auch in unserem Falle verfahren und daher die Lösung der salzsauren Salze nach dem Verdünnen mit Wasser auf etwa $150 \mathrm{ccm}$. mit einer gesättigten Lösung an Natriumpikrat so lange versetzt, als noch ein weiterer Zusatz des Fällungsmittels zu einem Theile des Filtrats sofort eine Trübung erzeugt. Der Niederschlag von pikrinsaurem Adenin wird sofort mit Hülfe einer Saugvorrichtung abfiltrirt.

Da Natriumpikrat aus der Lösung der Basen ausser Adenin auch Farbstoffe fällt, so kann das erhaltene Präparat nicht als reines Adeninpikrat angesehen werden. Um den Gehalt desselben an Adeninpikrat, resp. Adenin festzustellen,

1) Diese Zeitschrift, Bd. XXVI, S. 354. 
empfiehlt es sich, in einem abgewogenen Theile des lufttrockenen Präparates den Basengehalt mit Hülfe der Kupferoxydulfällung zu ermitteln und den Stickstoff dieser Fällung auf Adenin umzurechnen. Auf diese Weise ergab sich, dass der durch Natriumpikrat erzeugte Niederschlag 1,88 g Adenin enthielt.

Zur Reinigung wurde das Adeninpikrat in heissem Wasser unter Zusatz der berechneten Menge Normalnatronlauge gelöst, dann die der Lauge entsprechende Menge an Normalsalzsäure hinzugefügt und diese Lösung mit Thierkohle behandelt.

Aus dem Filtrate scheidet sich dann das Adeninpikrat in langen glänzenden Nadeln ab. Da von den natürlich vorkommenden Purinbasen nur vier, nämlich Guanin, Adenin, Hypoxanthin und Epiguanin Pikrate geben, welche sich zum Theil schon durch ihre Krystallform von einander unterscheiden, von denen ausserdem nur die des Adenins und Epiguanins bei bestimmten Temperaturen liegende Zersetzungspunkte haben, so ist zur Identitätsbestimmung des Adeninpikrates eine Analyse überflüssig, und es genügt die Ermittelung des Zersetzungspunktes. Derselbe lag bei unserem Präparate, verglichen mit dem einer anderen Herkunft, bei derselben Temperatur, $281^{\circ}$; auch ein Gemisch beider Präparate zeigte denselben Zersetzungspunkt.

Der Rest der neben Guanin und Adenin vorhandenen Basen wurde aus dem Filtrate von Adeninpikrat durch Kupfersulfat und Natriumbisulfit gefällt. Der gut ausgewaschene Niederschlag lieferte nach dem Zersetzen mit Schwefelwasserstoff ein Basengemisch in Gewichte von $0,438 \mathrm{~g}$, welches aus Xanthin und Hypoxanthin bestand.

Nach dem Entfärben durch Thierkohle wurde das Gemenge mit Salzsäure eingedampft und der Ueberschuss von Säure in der eben angegebenen Weise beseitigt. Die Lösung des Rückstandes, welcher neben den salzsauren Salzen freies Xanthin enthält, in wenig Wasser schied nach längerem Stehen 0,112 g Xanthin aus; daz Filtrat hinterliess nach dem Eindampfen 0,38g Substanz, hauptsächlich aus salzsaurem Hypoxanthin neben den Spuren in Lösung gebliebenen Xanthins bestehend. 
Da bei so geringen Mengen an Basen die Möglichkeit, dieselben, sei es in Form freier Basen, sei es in Form von Salzen, in zur Analyse hinreichender Ausbeute zu erhalten, durchaus nicht sicher gegeben ist, so empfiehlt es sich, die Körper zur Identificirung in solche Salze überzuführen, deren Löslichkeit und Krystallform für die einzelnen Basen charakteristisch sind und sie von den entsprechenden Salzen anderer Basen unterscheiden. Solche Salze sind beim Xanthin: das salpetersaure Salz und die Doppelverbindung mit Silbernitrat, beim Hypoxanthin : gleichfalls das salpetersaure Salz und fernerhin das Pikrat.

Das nach Vorschrift ${ }^{1}$ ) dargestellte Xanthinnitrat zeigte in unserem Falle die verlangten Krystallformen, ebenso das Doppelsalz mit Silbernitrat.

Das Hypoxanthin wurde zunächst mit Hülfe seines pikrinsauren Salzes gereinigt, weil bei Ueberführung in das salpetersaure Salz zu befürchten war, dass das noch beigemengte Xanthin durch die Salpetersäure gleichfalls mit niedergeschlagen würde. Ueberhaupt empfiehlt sich diese Abweichung von dem ursprünglichen Krüger-Salomon'schen Verfahren zur Trennung der Purinkörper in allen den Fällen, wo es sich, wie hier, um eine direkte Scheidung des Hypoxanthins und Xanthins handelt, weil dann die Gewissheit gegeben ist, dass das Hypoxanthin frei von Xanthin erhalten wird. Ausserdem gewährt die Ueberführung des Hypoxanthins in das Pikrat noch den Vortheil, dass die Spuren von Adenin, welche der Fällung mit Natriumpikrat entgangen sind, in bequemer Weise entfernt werden können.

Man verfährt zu dem Zwecke in folgender Weise: Das salzsaure Hypoxanthin, in unserem Falle $0,38 \mathrm{~g}$, wird zusammen mit einem kleinen Ueberschuss freier Pikrinsäure in etwa $50 \mathrm{ccm}$. heissen Wassers gelöst. Beim Abkühlen trübt sich die Lösung gleichmässig, wenn noch Adenin vorhanden ist. Die Trübung ballt sich beim Schütteln der Flüssigkeit leicht zu Flocken zusammen, welche sofort mit einer Saug-

1) 1. c., Bd. 26, S. 357 . 
vorrichtung abfiltrirt werden. Das klare Filtrat scheidet dann nach dem Einengen und Erkaltenlassen das Hypoxanthinpikrat in makroskopischen tafelförmigen Krystallen aus, welche mit keinem Pikrat einer anderen Purinbase $z u$ verwechseln sind. Zur Ueberführung in das salpetersaure Salz wird das Pikrat in Wasser unter Zusatz von Salpetersäure gelöst und die Pikrinsäure durch Ausschütteln mit Benzol oder Toluol entfernt. Beim Eindampfen der salpetersauren Lösung scheidet sich dann das Nitrat in wetzsteinförmigen Krystallen aus.

Als Resultat des vorliegenden Versuchs ergibt sich zunächst, dass unsere Versuchsperson im Laufe von 42 Tagen die folgenden Mengen an Nucleinbasen ausgeschieden hat: 2,363 g Guanin, 1,88 $\mathrm{g}$ Adenin, 0,112 $\mathrm{g}$ Xanthin und $0,300 \mathrm{~g}$ Hypoxanthin, in Summa $4,655 \mathrm{~g}$, oder pro Tag 0,110 g Basen. Diese 0,11 g Basen enthalten 0,0532 g Basenstickstoff. Da bei derselben Versuchsperson auch der mit dem Urine entleerte Basenstickstoff von M. Krüger und J. Schmid ${ }^{1}$ ) bestimmt ist, dessen Menge pro Tag 16,6 mg betrug, so ist ein Vergleich beider Ausscheidungsgrössen möglich; er ergibt, dass mit dem Kothe mehr als die dreifache Menge an Basen ausgeschieden wird, wie mit dem Harne.

Es mag hier sogleich erwähnt werden, was erst in einer späteren Publication ausführlicher mitgetheilt werden soll, dass die Art der Basen und die Zusammensetzung des Basengemisches für Harn und Koth durchaus verschieden sind.

Die obere Grenze der pro Tag mit den Faeces ausgeschiedenen Basenmenge, welche Petrén mit $0,1 \mathrm{~g}$ annimmt, ist nach unserem Versuche jedenfalls $\mathrm{zu}$ niedrig angegeben. Auch die von ihm herrührende Beobachtung, dass die Xanthinund Hypoxanthinfraction annähernd gleich sind, entspricht in keiner Weise den Thatsachen. Vielmehr übertrifft die Hypoxanthinfraction, $\mathrm{zu}$ welcher Hypoxanthin; Adenin und Guanin gehören, die Xanthinfraction um ein Vielfaches. Der Irrthum

1) Diese Zeitschrift, Bd. XXXII, S. 107. 
Petrén's findet seine Erklärung in der Anwendung des Neubauer'schen Verfahrens zur Trennung der Purinkörper, bei welchem durch Umwandlung von Guanin in Xanthin die Xanthinfraction auf Kosten der Hypoxanthinfraction bereichert werden muss.

Als Hauptergebniss unseres Versuches ist jedoch die Auffindung des Adenins und die Beobachtung, dass Adenin in Gemeinschaft mit Guanin bei Weitem den grössten Antheil zu den Kothbasen liefert, anzusehen.

Dies Resultat ist ein völlig anderes, als es nach den Untersuchungen von Schindler erwartet werden sollte. Die einfachste Erklärung würde sein, dass die Darmfäulniss nicht dieselbe Wirkung auf Adenin und Guanin hat, wie die von Schindler bei seinen Versuchen angewendete und durch Pankreasinfus hervorgerufene Fäulniss.

Wenn trotzdem aber die Darmfäulniss in derselben Weise wie die Pankreasfäulniss Guanin in Xanthin und Adenin in Hypoxanthin umwandelt, so würde die Zusammensetzung des Basengemisches, wie wir sie im Kothe gefunden haben, nur dadurch zu Stande kommen können, dass die Fäulnissprodukte, Xanthin und Hypoxanthin, bei Weitem leichter resorbirt werden, als Guanin und Adenin, so dass der der Umwandlung entgangene Rest der letzteren Basen schliesslich doch die Hauptmenge der mit dem Kothe entleerten Basen ausmachen könnte.

Nun wird aber das schwerlösliche Xanthin zweifellos bedeutend langsamer und unvollständiger resorbirt, als Adenin. Es muss also eine im Sinne der Pankreasfäulniss verlaufende Darmfäulniss offenbar ein Basengemisch von anderer Zusammensetzung erzeugen, als es in unserem Versuche vorlag, bei dem die gesammten, sowohl bacteriellen, als durch Fermente bedingten Fäulnissprocesse des menschlichen Darmes in Betracht kommen.

Wir sind im Begriffe, diese Frage im Anschlusse an die Versuche Schindler's unter gleichzeitiger Kontrolle der bekannten chemischen Produkte der Fäulniss und mit menschlichen Faeces verschiedener Herkunft weiter zu studiren. 